\title{
Investigation of the predictive value of speckle tracking imaging for the assessment of cardiac function and clinical prognosis in patients with hypercholesterolemia
}

\author{
LING ZHANG and XIAOXU CHEN \\ Department of Physical Diagnosis, Daqing Longnan Hospital, Daqing, Heilongjiang 163453, P.R. China
}

Received September 14, 2016; Accepted March 17, 2017

DOI: $10.3892 /$ etm.2017.4325

\begin{abstract}
The predictive value of speckle tracking imaging (STI) for the assessment of cardiac function and clinical prognosis in patients with hypercholesterolemia was explored. One hundred forty-seven patients with hypercholesterolemia diagnosed in our hospital and 137 healthy adults from our health examination center over the same period were included in this study. Using STI and color Doppler ultrasound, we carried out follow-up of cardiac function in patients with hypercholesterolemia and statistical analysis based on collected data. Global longitudinal strain, global circumferential strain, and the strains of apical long-axis, four-chamber and two-chamber planes, and short-axis mitral valve, short-axis papillary muscle, and short-axis apical levels in the hypercholesterolemia group were significantly lower than those in the control group. Global systolic and early diastolic strain rates were significantly lower than those in the control group. In the coronaries, the percentage of segments with significant differences was $80 \%(24 / 30)$ in the left anterior descending coronary artery (LAD), 66.7\% (9/12) in the left circumflex coronary artery (LCX), and 75\% (8/12) in the right coronary artery (RCA). The average strains in the long axis, radial, and circumferential directions (Strain ${ }_{\mathrm{SL}, \mathrm{SC}, \mathrm{SR}}-\mathrm{Avg}$ ) in the three main coronaries were significantly different between the hypercholesterolemia group and control group $(\mathrm{P}<0.0001)$. In the three coronaries and their basal and mid segments, there were significant differences between LCX and LAD, and between LCX and RCA $(\mathrm{P}<0.0001)$. In conclusion, the application of STI for monitoring cardiac function in patients with hypercholesterolemia provides physicians with useful information related to hypercholesterolemia to achieve early diagnosis and effective treatment.
\end{abstract}

Correspondence to: Dr Xiaoxu Chen, Department of Physical Diagnosis, Daqing Longnan Hospital, 35 Aiguo Road, Daqing, Heilongjiang 163453, P.R. China

E-mail: chen_xiaoxu1212@163.com

Key words: hypercholesterolemia, cardiac function, speckle tracking imaging

\section{Introduction}

Speckle tracking imaging (STI) identifies and tracks the ultrasonic speckle motion and relative motion between speckles scattered in myocardial tissue, frame by frame in ultrasonic images. As a new method of ultrasonic assessment of cardiac function, it can rapidly quantify the strain value of each myocardial segment, which is unaffected by the swing and pull of the heart (1). In physics, strain (S) is the relative deformation of an object under an external force. Strain rate (SR) is the speed at which the deformation of the object occurs. Myocardial strain refers to myocardial deformation during the cardiac cycle, which can be used for the assessment of parameters such as local systolic and diastolic myocardial function, the state of blood supply, and myocardial viability. Myocardial strain rate is the speed at which myocardial deformation occurs. Myocardial strain and strain rate reflect the degree and speed of myocardial deformation, respectively, and can be used for the assessment of local and global systolic and diastolic myocardial functions (2). Assessing early coronary and aortic atherosclerotic processes and the function of coronary circulation in patients with hypercholesterolemia are key to intervention. STI can provide information on local and global myocardial structures and functions. Strain rate and related speed data can be derived from information on strain, which is helpful for the early detection of diseases caused by left ventricular (LV) dysfunction (3).

Traditional transthoracic Doppler echocardiography (TTDE) can only detect wall motion abnormalities, valve changes, or abnormal cardiac function when electrocardiographicchanges arefound in patients with hypercholesterolemia. In this study, we compared it with traditional TTDE (4,5). As a novel and sensitive echocardiography technique, STI allows for early detection of cardiac abnormalities in patients with hypercholesterolemia and normal ejection fraction (EF). It can be used to make preliminary analysis of clinical prognosis for the patients.

\section{Patients and methods}

Patients with hypercholesterolemia. A total of 147 patients with hypercholesterolemia diagnosed in the division of cardiovascular medicine of our hospital between August 2010 and 
July 2015 were selected, including 80 males and 67 females. Their ages ranged from 35 to 79 years, with average age of $51.3 \pm 14.6$ years.

Clinical diagnosis of hypercholesterolemia was based on previous evidence (6): i) Serum total cholesterol (TC) in adults $>7.8 \mathrm{mmol} / \mathrm{l}(300 \mathrm{mg} / \mathrm{dl})$, TC in children under 16 years $>6.7 \mathrm{mmol} / \mathrm{l}(260 \mathrm{mg} / \mathrm{dl})$ or serum low-density lipoprotein cholesterol (LCL-c) $>4.9 \mathrm{mmol} / \mathrm{l}(190 \mathrm{mg} / \mathrm{dl})$; ii) patients or their relatives have xanthoma of the skin or tendons; iii) TC $\geq 16 \mathrm{mmol} / \mathrm{l}(600 \mathrm{mg} / \mathrm{dl})$.

Fasting venous blood was drawn from patients to measure serum TC, LDL-c, triglyceride (TG), and high-density lipoprotein cholesterol (HDL-c).

All patients were examined by echocardiography to confirm that EF was normal. In addition, several other parameters were recorded, including normal blood pressure. Exclusion criteria were: Smoking history, vascular diseases, medicine treatment, liver or kidney dysfunction, abnormality of blood calcium or glucometabolism, hypertrophic cardiomyopathy or rheumatic heart valve disease, and diseases causing secondary dyslipidemia such as pancreatitis, myeloma, Cushing's syndrome, hyperthyroidism or hypothyroidism, hypopituitarism and systemic lupus erythematosus.

Control group. The healthy control group included 137 subjects: 81 males and 56 females, aged from 32 to 76 years, with average age of $52.3 \pm 13.4$ years. All subjects in the control group were normal according to physical examination, electrocardiography, and echocardiography. Furthermore, they met all the following criteria: No history of heart diseases, absence of liver or kidney dysfunction, absence of abnormality of blood calcium or glucometabolism, absence of diseases causing secondary dyslipidemia, and absence of hypertrophic cardiomyopathy or rheumatic heart valve disease. The study was approved by the Local Ethics Committee, and all participants signed the informed consent.

Instruments. A Vivid 7 dimension color Doppler ultrasound diagnostic system from GE Healthcare (Piscataway, NJ, USA) was used. Active matrix array probes (M4S and M3S) were used with frequency ranging from 1.7 to $3.4 \mathrm{MHz}$, equipped with 2-dimensional STI (2D-STI) analysis software (EchoPAC 7.0 workstation).

Conventional echocardiographic examination. For conventional echocardiographic examination, participants were made to lie in the left-lateral decubitus position and were synchronously connected to the electrocardiography machine. For 2-dimensional echocardiography, we selected the parasternal long axis plane, mitral valve level, papillary muscle level, apical level short axis planes, and apical four-chamber $(4 \mathrm{CH})$ and two-chamber $(2 \mathrm{CH})$ planes to observe cardiac morphology, size of the cardiac chamber, thickness of chamber wall and motion, the morphology and the opening and closing movements of the valves, thickness of vessel wall and the lumen of the aortic root and proximal ascending aorta. M-type echocardiography was used to measure LV cavity size and calculate cardiac function, including EF and fractional shortening. The modified Simpson's method was used to measure EF when regional wall motion abnormalities occurred. Color Doppler was applied to observe the cardiac chambers, aortic blood flow, and valve regurgitation. Regurgitation area was used to quantitatively assess the degree of regurgitation. Using pulsed continuous-wave Doppler, the blood flow velocity across each valve orifice was measured, including maximum aortic velocity across the aortic valve orifice (AOVmax), pressure gradient (PG), frequency spectrum $\mathrm{E}$ peak, A peak, the ratio of E/A across the mitral valve orifice, and early diastolic deceleration time (DT). Using tissue Doppler imaging, the early diastolic mitral annulus velocity, e', and the ratio of E/e' were measured. All results were the average values of three cardiac cycles.

2D-STI image acquisition and analysis. Parasternal B-mode second-harmonic imaging was used to acquire the views of the apical long-axis (APLAX), 4CH, 2CH, short-axis mitral valve (SAX-MV), short-axis papillary muscle (SAX-PM), and short-axis apical (SAX-AP) levels, which were stored and analyzed offline. The frame rate ranged from 40 to 80 frames per second. Initially, the APLAX plane of one cardiac period was selected. The region of interest between the endocardium and epicardium was drawn automatically or manually in the LV endocardial boundary at the end of systole. Subsequently, the workstation calculated and generated color strain curves, and automatically divided the LV plane view into six sections. $4 \mathrm{CH}$ (Fig. 1A) and 2CH planes were applied for the same operation. The naming of the segments originated from the LV segment nomenclature issued by the American Society of Echocardiography (ASE): Six segments were divided into parasternal long axis and short axis planes: The anterior wall, anterior septum, lateral wall, posterior wall, basal segments of the lower wall and posterior septum, mid-segments, and apical segments. Based on the analysis of strain curves of three cardiac cycles, the long axis, circumferential, and radial strain values (SL, SC, and SR) were derived. The program software was used to generate 18 segmental strains and the average values were used as the global longitudinal strain (GLS) (Fig. 1C), global circumferential strain (GCS) (Fig. 1D), and global radial strain (GRS) (Fig. 1E). Strain rate is the speed at which myocardial strain occurs (Fig. 1B). Global systolic strain rate (GSRs), global early and late diastolic strain rates (GSRe and GSRa), and the strain rates of APLAX, $4 \mathrm{CH}$ and $2 \mathrm{CH}$ planes, and SAX-MV, SAX-PM, and SAX-AP levels were obtained, respectively. Based on the average strain values of deformed myocardial segments and main coronary arteries in three directions $\left(\right.$ Strain $_{\mathrm{SL}, \mathrm{SC}, \mathrm{SR}}-\mathrm{Avg}$ ), we identified the coronary artery that underwent the largest deformation or underwent the most severe damage.

Statistical analysis. Data were analyzed with SPSS 16.0 statistical software. Normally distributed data were analyzed by independent-sample t-test, and expressed as mean \pm standard deviation; abnormally distributed data were analyzed by the Mann-Whitney U test, and expressed as median (P2.5-P97.5). These two data sets were analyzed by the Kruskal-Wallis test. Linear regression analysis was used to study the correlation between LDL-c and EF, GLS, GCS, GRS, GSRs, GSRe, and GSRa, respectively. $\mathrm{P}<0.05$ was considered statistically significant. Inter-group and intra-group differences were analyzed by Pearson's correlation between two variables. 


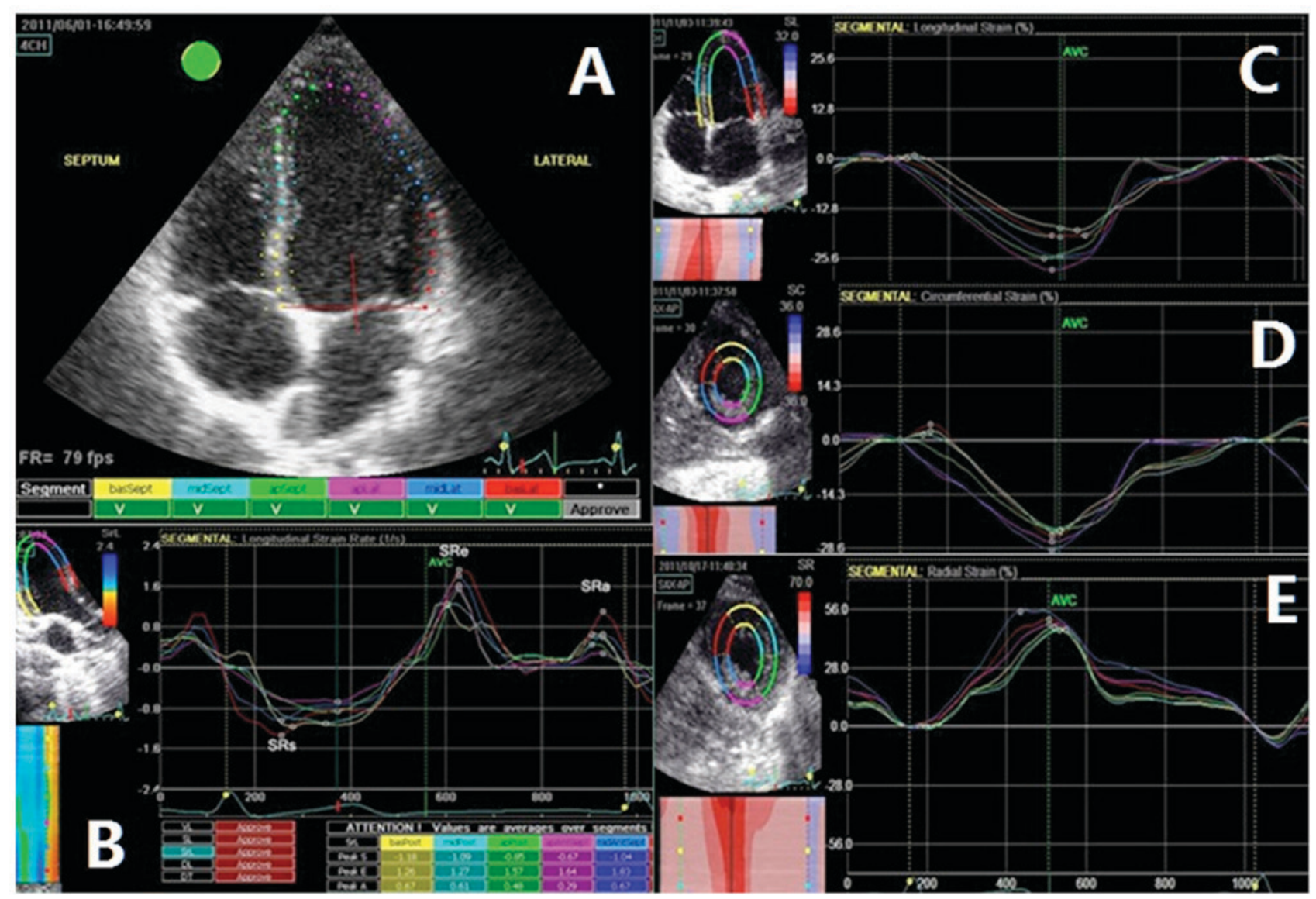

Figure 1. Two-dimensional speckle tracking imaging (STI) analysis system. (A) The region of interest between the endocardium and epicardium drawn automatically or manually in the left ventricular endocardial boundary at the end of systole. (B) Global systolic strain rate (GSRs) and global early and late diastolic strain rates (GSRe and GSRa). (C) Global long axis strain (GLS). (D) Global circumferential strain (GCS). (E) Global radial strain (GRS).

Table I. Comparison of general parameters between the hypercholesterolemia and control groups.

\begin{tabular}{lccc}
\hline Variables & Hypercholesterolemia group $(\mathrm{n}=147)$ & Control group $(\mathrm{n}=137)$ & P-value \\
\hline Age (years) & $21.7 \pm 14.2$ & $21.8 \pm 13.4$ & \\
& (range, $4-49$ years) & (range, 2-46 years) & 0.96 \\
Male $(\%)$ & $58.3 \%$ & $56.7 \%$ & 0.93 \\
TC & $13.47 \pm 5.06$ & $5.32 \pm 1.05$ & $<0.0001$ \\
LDL-c (mmol/l) & $10.84 \pm 4.76$ & $3.27 \pm 0.77$ & $<0.0001$ \\
TG (mmol/l) & $1.66 \pm 0.63$ & $1.41 \pm 0.36$ & 0.32 \\
HDL-c (mmol/l) & $1.53 \pm 0.74$ & $1.64 \pm 0.67$ & 0.49 \\
Systolic pressure $(\mathrm{mmHg})$ & $108.85 \pm 16.81$ & $108.11 \pm 9.83$ & 0.81 \\
Diastolic pressure (mmHg) & $64.94 \pm 9.99$ & $64.97 \pm 5.74$ & 0.95 \\
Heart rate (bpm) & $80.38 \pm 14.42$ & $79.97 \pm 9.49$ & 0.88 \\
\hline
\end{tabular}

TC, total cholesterol; LDL-c, low-density lipoprotein cholesterol; TG, triglyceride; HDL-c, high-density lipoprotein cholesterol.

\section{Results}

General parameters. The general parameters of subjects in the two groups are shown in Table I. There were no significant differences in age, gender, blood pressure, and heart rate between the two groups. The serum TC and LCL-c of patients in the hypercholesterolemia group were significantly higher than those in the control group $(13.47 \pm 5.06$ vs. $5.32 \pm 1.05$,
$10.84 \pm 4.76$ vs. $3.27 \pm 0.77, \mathrm{P}<0.0001)$. There were no significant differences in HCL-c and TG between the two groups: $1.53 \pm 0.74$ vs. $1.64 \pm 0.67, \mathrm{P}=0.49$ and $1.66 \pm 0.63$ vs. $1.41 \pm 0.36$, $\mathrm{P}=0.32$.

Conventional echocardiography. Results showed that the left ventricle enlarged and the chamber wall thickened in patients with hypercholesterolemia. There were no significant changes 
Table II. Comparison of conventional echocardiography examinations between the hypercholesterolemia and control groups.

\begin{tabular}{|c|c|c|c|}
\hline Variables & Hypercholesterolemia group $(n=47)$ & Control group $(n=37)$ & P-value \\
\hline LV diastolic diameter (mm) & $44.39 \pm 7.67$ & $40.81 \pm 6.01$ & 0.02 \\
\hline LV systolic diameter (mm) & $29.59 \pm 6.59$ & $26.38 \pm 4.51$ & 0.013 \\
\hline Ventricular septum (mm) & $8.25 \pm 1.26$ & $7.14 \pm 0.98$ & $<0.0001$ \\
\hline LV posterior wall (mm) & $8.26 \pm 1.35$ & $6.91 \pm 1.16$ & $<0.0001$ \\
\hline Maximum aortic velocity (AOVmax) & $197.04 \pm 81.91$ & $123.62 \pm 12.28$ & $<0.0001$ \\
\hline Aortic pressure gradient $(\mathrm{PG}, \mathrm{mmHg})$ & $18.21 \pm 15.53$ & $6.30 \pm 1.13$ & $<0.0001$ \\
\hline LV EF (\%) & $64.28 \pm 8.13$ & $65.70 \pm 2.58$ & 0.87 \\
\hline E peak & $99.06 \pm 21.65$ & $101.22 \pm 13.81$ & 0.59 \\
\hline A peak & $56.67 \pm 12.56$ & $55.89 \pm 12.93$ & 0.78 \\
\hline $\mathrm{E} / \mathrm{A}$ & $1.69 \pm 0.45$ & $1.76 \pm 0.33$ & 0.46 \\
\hline $\mathrm{E} / \mathrm{e}^{\prime}$ & $7.8 \pm 2.3$ & $5.2 \pm 1.9$ & 0.006 \\
\hline DT (msec) & $183 \pm 45$ & $170 \pm 40$ & 0.001 \\
\hline
\end{tabular}

LV, left ventricular; EF, ejection fraction; DT, deceleration time.

Table III. Comparison of global strain between the hypercholesterolemia and control groups.

\begin{tabular}{lccc}
\hline $\begin{array}{l}\text { Global } \\
\text { strain }(\%)\end{array}$ & $\begin{array}{c}\text { Hypercholesterolemia } \\
\text { group }(\mathrm{n}=47)\end{array}$ & $\begin{array}{c}\text { Control } \\
\text { group }(\mathrm{n}=37)\end{array}$ & P-value \\
\hline GLS & $-19.86 \pm 4.76$ & $-24.44 \pm 1.73$ & $<0.0001$ \\
GCS & $-20.95 \pm 5.21$ & $-25.92 \pm 2.78$ & $<0.0001$ \\
GRS & $52.64 \pm 14.85$ & $41.76 \pm 14.82$ & 0.006 \\
APLAX & $-19.6 \pm 5.49$ & $-24.8 \pm 2.65$ & $<0.0001$ \\
4CH & $-19.08 \pm 5.08$ & $-23.89 \pm 2.42$ & $<0.0001$ \\
2CH & $-20.64 \pm 5.39$ & $-24.69 \pm 2.4$ & 0.001 \\
SAX-MV & $-17.7 \pm 5.07$ & $-24.06 \pm 3.36$ & $<0.0001$ \\
SAX-PM & $-20.27 \pm 6.42$ & $-25.78 \pm 4.27$ & $<0.0001$ \\
SAX-AP & $-24.57 \pm 7.13$ & $-28.02 \pm 4.79$ & 0.013 \\
\hline
\end{tabular}

GLS, global longitudinal strain; GCS, global circumferential strain; GRS, global radial strain; APLAX, apical long-axis; $4 \mathrm{CH}$, four-chamber plane; $2 \mathrm{CH}$, two-chamber plane; SAX-MV, short-axis mitral valve level; SAX-PM, short-axis papillary muscle level; SAX-AP, short-axis apical level.

in EF between the two groups $(64.28 \pm 8.13$ vs. $65.70 \pm 2.58$, $\mathrm{P}=0.87$ ). The maximum aortic velocity and pressure gradient were significantly higher than those in the control group $(197.04 \pm 81.91$ vs. $123.62 \pm 12.28,18.21 \pm 15.53$ vs. $6.30 \pm 1.1$, $\mathrm{P}<0.0001)$. In the assessment of diastolic function, there were no significant differences in E peak, A peak, and E/A between the two groups, while E/e' and DT in the hypercholesterolemia group were significantly higher than those in the control group $(7.8 \pm 2.3$ vs. $5.2 \pm 1.9, \mathrm{P}=0.006,183 \pm 45$ vs. $170 \pm 40$, $\mathrm{P}=0.001)$ (Table II).

2D-STI. GLS, GCS, and the strains of APLAX, 4CH and 2CH planes, and SAX-MV, SAX-PM, and SAX-AP levels in the hypercholesterolemia group were significantly lower than those in the control group except for GRS, which
Table IV. Comparison of GSRs between the hypercholesterolemia and control groups.

\begin{tabular}{lccc}
\hline $\begin{array}{l}\text { Strain rate } \\
(1 / \mathrm{sec})\end{array}$ & $\begin{array}{c}\text { Hypercholesterolemia } \\
\text { group }(\mathrm{n}=47)\end{array}$ & $\begin{array}{c}\text { Control } \\
\text { group }(\mathrm{n}=37)\end{array}$ & P-value \\
\hline GSRs & $-1.18 \pm 0.29$ & $-1.42 \pm 0.24$ & 0.02 \\
GSRe & $1.68 \pm 0.50$ & $2.13 \pm 0.48$ & 0.001 \\
GSRa & $0.82 \pm 0.35$ & $0.76 \pm 0.25$ & 0.78 \\
\hline
\end{tabular}

GSRs, global systolic strain rate; GSRe, global early diastolic strain rate; GSRa, global late diastolic strain rate.

increased (Table III). GSRs and GSRe were significantly lower than those in the control group $(-1.18 \pm 0.29$ vs. $-1.42 \pm 0.24$, $\mathrm{P}=0.01,1.68 \pm 0.50$ vs. $2.13 \pm 0.48, \mathrm{P}=0.0001)$ (Table IV). There were no significant differences between the two groups in GSRa and SRa of the LV segmental wall, and SRs and SRe of SAX-AP (Table V). The long axis and circumferential strains of patients with hypercholesterolemia were significantly decreased, while the radial strain increased significantly. According to the ASE guided distribution areas of the main coronaries, the strain values in three directions are listed in Tables VI-VIII. LCL-c was significantly associated with GLS, GCS, and GRS ( $\mathrm{r}=0.561, \mathrm{P}=0.001 ; \mathrm{r}=0.565, \mathrm{P}<0.0001 ; \mathrm{r}=0.563$, $\mathrm{P}=0.003$ ) (Figs. 2-4), but not with EF, GSRs, GSRa, and GSRe. In the coronaries, the percentage of segments with significant differences was $80 \%(24 / 30)$ in the left anterior descending coronary artery (LAD), $66.7 \%$ (9/12) in left circumflex coronary artery (LCX), and $75 \%(8 / 12)$ in right coronary artery (RCA) (Table IX). The average strains in the long axis, radial, and circumferential directions ( Strain $_{\mathrm{SL}, \mathrm{SC}, \mathrm{SR}}-\mathrm{Avg}$ ) in the three main coronaries were significantly different between the hypercholesterolemia and control groups $(\mathrm{P}<0.0001)$. Pairwise comparisons showed that in the three coronaries and their basal and mid-segments, there were significant differences between LCX and LAD, and between LCX and RCA $(\mathrm{P}<0.0001)$. The 
Table V. Comparison of plane strain rate between the hypercholesterolemia and control groups.

\begin{tabular}{lccc}
\hline $\begin{array}{l}\text { Strain rate } \\
(1 / \mathrm{sec})\end{array}$ & $\begin{array}{c}\text { Hypercholesterolemia } \\
\text { group }(\mathrm{n}=47)\end{array}$ & $\begin{array}{c}\text { Control } \\
\text { group }(\mathrm{n}=37)\end{array}$ & $\begin{array}{c}\text { P-value } \\
\text { SRs }\end{array}$ \\
APLAX & $-1.11 \pm 0.36$ & $-1.36 \pm 0.22$ & $<0.0001$ \\
4CH & $-1.04 \pm 0.29$ & $-1.25 \pm 0.21$ & $<0.0001$ \\
2CH & $-1.10 \pm 0.32$ & $-1.28 \pm 0.18$ & 0.004 \\
SAX-MV & $-1.14 \pm 0.32$ & $-1.42 \pm 0.26$ & $<0.0001$ \\
SAX-PM & $-1.20 \pm 0.42$ & $-1.53 \pm 0.33$ & $<0.0001$ \\
SAX-AP & $-1.57 \pm 0.61$ & $-1.77 \pm 0.41$ & 0.095 \\
SRe & & & \\
APLAX & $1.51 \pm 0.68$ & $2.03 \pm 0.52$ & $<0.0001$ \\
4CH & $1.63 \pm 0.74$ & $2.12 \pm 0.56$ & 0.001 \\
2CH & $1.58 \pm 0.65$ & $1.98 \pm 0.42$ & 0.001 \\
SAX-MV & $1.43 \pm 0.63$ & $2.02 \pm 0.47$ & $<0.0001$ \\
SAX-PM & $1.73 \pm 0.76$ & $2.17 \pm 0.70$ & 0.008 \\
SAX-AP & $2.15 \pm 1.02$ & $2.51 \pm 0.70$ & 0.069 \\
SRa & & & \\
APLAX & $0.88 \pm 0.31$ & $0.79 \pm 0.30$ & 0.21 \\
4CH & $0.84 \pm 0.21$ & $0.83 \pm 0.29$ & 0.87 \\
2CH & $0.85 \pm 0.26$ & $0.78 \pm 0.29$ & 0.22 \\
SAX-MV & $0.65 \pm 0.44$ & $0.56 \pm 0.38$ & 0.29 \\
SAX-PM & $0.70 \pm 0.43$ & $0.66 \pm 0.52$ & 0.71 \\
SAX-AP & $0.88 \pm 0.57$ & $0.79 \pm 0.59$ & 0.47 \\
\hline
\end{tabular}

SR, strain rate; SRe, early strain rate; SRa, late strain rate; APLAX, apical long-axis; $4 \mathrm{CH}$, four-chamber plane; $2 \mathrm{CH}$, two-chamber plane; SAX-MV, short-axis mitral valve level; SAX-PM, short-axis papillary muscle level; SAX-AP, short-axis apical level.

average strains of LAD, LCX, and RCA in the hypercholesterolemia and control groups were $-17.15(-38.12-72.14)$ vs. -22.97 (-40.35-69.12), -10.41 (-29.41-75.9) vs. -16.13 (-31.44-81.12), and -17.39 (-36.22-77.25) vs. -20.91 (-41.45-76.63), respectively [abnormal distribution, expressed as median (P2.5-P97.5), Kruskal-Wallis test] (Table X).

\section{Discussion}

Hypercholesterolemia is highly prevalent (5). Worldwide, there are $\sim 140,000,000$ patients with hypercholesterolemia, and $\sim 26,000,000$ potential patients with the disease in China (7). In addition, the data from the Atherosclerosis Society that was collected in Hong Kong, Taiwan, and China mainland are not included (8). According to the assessment by the World Health Organization, the percentage of patients diagnosed with hypercholesterolemia is $<25 \%$ (9). However, the diagnosis of hypercholesterolemia is often made after the occurrence of severe cardiovascular events. Therefore, the most effective measure to prevent morbidity and mortality is early diagnosis and treatment.

2D-STI has been widely used to assess cardiac functions. Myocardial strain and strain rate reflect the degree and speed of myocardial deformation, respectively. It can be used in the
Table VI. Comparison of long axis strain (SL) between the hypercholesterolemia and control groups.

\begin{tabular}{|c|c|c|c|}
\hline SL strain $(\%)$ & $\begin{array}{l}\text { Hypercho- } \\
\text { lesterolemia } \\
\text { group }(n=47)\end{array}$ & $\begin{array}{l}\text { Control } \\
\text { group } \\
(n=37)\end{array}$ & P-value \\
\hline \multicolumn{4}{|l|}{$\begin{array}{l}\text { LAD A3C } \\
\text { anterior septum }\end{array}$} \\
\hline Basal segment & $-18.93 \pm 5.52$ & $-22.27 \pm 3.15$ & 0.001 \\
\hline Mid-segment & $-21.84 \pm 6.15$ & $-25.73 \pm 3.82$ & 0.001 \\
\hline Apical segment & $-24.3 \pm 8.4$ & $-29.1 \pm 5.6$ & 0.002 \\
\hline \multicolumn{4}{|l|}{$\mathrm{A} 3 \mathrm{C}$ posterior wall } \\
\hline Apical segment & $-21.8 \pm 8.3$ & $-26.8 \pm 5.2$ & 0.001 \\
\hline \multicolumn{4}{|l|}{$\mathrm{A} 2 \mathrm{C}$ anterior wall } \\
\hline Basal segment & $-17.76 \pm 7.67$ & $-21.48 \pm 7.69$ & 0.006 \\
\hline Mid-segment & $-19.21 \pm 6.42$ & $-25.33 \pm 3.24$ & $<0.0001$ \\
\hline Apical segment & $-21.95 \pm 8.66$ & $-28.17 \pm 4.18$ & $<0.0001$ \\
\hline \multicolumn{4}{|l|}{ A2C lower wall } \\
\hline Apical segment & $-24.49 \pm 8.11$ & $-28.62 \pm 3.63$ & 0.002 \\
\hline \multicolumn{4}{|l|}{$\begin{array}{l}\text { A } 4 \text { C posterior } \\
\text { septum }\end{array}$} \\
\hline Apical segment & $-22.94 \pm 5.45$ & $-27.56 \pm 4.57$ & $<0.0001$ \\
\hline \multicolumn{4}{|l|}{ A4C lateral wall } \\
\hline Apical segment & $-24.49 \pm 8.11$ & $-28.62 \pm 3.63$ & 0.002 \\
\hline \multicolumn{4}{|l|}{$\begin{array}{l}\text { LCX A3C } \\
\text { posterior wall }\end{array}$} \\
\hline Basal segment & $-17.83 \pm 6.74$ & $-23.85 \pm 3.76$ & $<0.0001$ \\
\hline Mid-segment & $-18.97 \pm 7.58$ & $-24.49 \pm 4.11$ & $<0.0001$ \\
\hline \multicolumn{4}{|l|}{ A4C lateral wall } \\
\hline Basal segment & $-17.82 \pm 6.73$ & $-23.84 \pm 3.75$ & $<0.0001$ \\
\hline Mid-segment & $-18.96 \pm 7.57$ & $-24.48 \pm 4.19$ & $<0.0001$ \\
\hline \multicolumn{4}{|l|}{$\begin{array}{l}\text { RCA A2C } \\
\text { lower wall }\end{array}$} \\
\hline Basal segment & $-21.59 \pm 5.61$ & $-23.72 \pm 3.83$ & 0.038 \\
\hline Mid-segment & $-22.24 \pm 5.55$ & $-24.86 \pm 3.17$ & 0.008 \\
\hline \multicolumn{4}{|l|}{$\begin{array}{l}\text { A4C posterior } \\
\text { septum }\end{array}$} \\
\hline Basal segment & $-17.61 \pm 4.82$ & $-20.73 \pm 3.14$ & 0.001 \\
\hline Mid-segment & $-20.15 \pm 4.56$ & $-23.47 \pm 2.18$ & $<0.0001$ \\
\hline
\end{tabular}

LAD, left anterior descending coronary artery; LCX, left circumflex coronary artery; RCA, right coronary artery.

assessment of local and global systolic and diastolic functions, as well as in the assessment of early LV functional abnormalities in patients with functional abnormalities of the heart, penetrating and non-penetrating myocardial infarction, heart valve damage, and other systemic diseases. STI can also detect early subclinical myocardial abnormalities in patients with diabetes and hypertensive heart disease (10), cardiac amyloidosis (11), ataxia (12), cardiac toxicity or radiotherapy (13), and rheumatic heart disease (14).

This study demonstrated that the ultrasonic technique, STI, can detect subclinical abnormalities of both systolic and 
Table VII. Comparison of circumferential strain (SC) between the hypercholesterolemia and control groups.

\begin{tabular}{|c|c|c|c|}
\hline SC strain (\%) & $\begin{array}{l}\text { Hypercho- } \\
\text { lesterolemia } \\
\text { group }(n=47)\end{array}$ & $\begin{array}{l}\text { Control } \\
\text { group }(n=37)\end{array}$ & P-value \\
\hline \multicolumn{4}{|l|}{$\begin{array}{l}\text { LAD mitral valve } \\
\text { level }\end{array}$} \\
\hline Anterior septum & $-27.11 \pm 8.22$ & $-33.53 \pm 5.14$ & $<0.0001$ \\
\hline Anterior wall & $-16.85 \pm 8.76$ & $-22.57 \pm 5.58$ & $<0.0001$ \\
\hline \multicolumn{4}{|l|}{$\begin{array}{l}\text { Papillary muscle } \\
\text { level }\end{array}$} \\
\hline Anterior septum & $-27.29 \pm 10.31$ & $-33.62 \pm 6.13$ & 0.001 \\
\hline Anterior wall & $-20.14 \pm 8.75$ & $-25.46 \pm 6.67$ & 0.002 \\
\hline \multicolumn{4}{|l|}{ Apical level } \\
\hline Anterior septum & $-25.38 \pm 11.29$ & $-31.41 \pm 5.52$ & 0.002 \\
\hline Anterior wall & $-24.33 \pm 8.54$ & $-26.55 \pm 6.76$ & 0.17 \\
\hline Lateral wall & $-22.87 \pm 9.78$ & $-24.19 \pm 8.41$ & 0.52 \\
\hline Posterior wall & $-22.92 \pm 7.93$ & $-24.84 \pm 8.45$ & 0.29 \\
\hline Lower wall & $-23.96 \pm 7.17$ & $-26.98 \pm 8.39$ & 0.08 \\
\hline Posterior septum & $-25.21 \pm 11.27$ & $-34.13 \pm 6.14$ & $<0.0001$ \\
\hline \multicolumn{4}{|l|}{$\begin{array}{l}\text { LCX mitral valve } \\
\text { level }\end{array}$} \\
\hline Lateral wall & $-18.25 \pm 7.66$ & $-24.37 \pm 7.68$ & $<.0001$ \\
\hline Posterior wall & $-23.29 \pm 8.21$ & $-26.62 \pm 8.53$ & 0.002 \\
\hline \multicolumn{4}{|l|}{$\begin{array}{l}\text { Papillary muscle } \\
\text { level }\end{array}$} \\
\hline Lateral wall & $-22.35 \pm 8.26$ & $-26.87 \pm 7.58$ & 0.009 \\
\hline Posterior wall & $-23.29 \pm 8.21$ & $-26.62 \pm 8.53$ & 0.06 \\
\hline \multicolumn{4}{|l|}{$\begin{array}{l}\text { RCA mitral valve } \\
\text { level }\end{array}$} \\
\hline Lower wall & $-16.54 \pm 7.95$ & $-20.66 \pm 6.47$ & 0.01 \\
\hline Posterior septum & $-27.88 \pm 7.19$ & $-30.11 \pm 5.52$ & $<0.0001$ \\
\hline \multicolumn{4}{|l|}{$\begin{array}{l}\text { Papillary muscle } \\
\text { level }\end{array}$} \\
\hline Lower wall & $-19.13 \pm 7.34$ & $-23.25 \pm 5.66$ & 0.004 \\
\hline Posterior septum & $-28.77 \pm 8.58$ & $-33.19 \pm 6.31$ & $<0.0001$ \\
\hline
\end{tabular}

LAD, left anterior descending coronary artery; LCX, left circumflex coronary artery; RCA, right coronary artery.

diastolic functions in the diagnosis of hypercholesterolemia. Although the parameters of conventional echocardiography showed that the LV wall thickened abnormally and the aorta narrowed, the normal wall motion and EF maintained normal systolic function. Data showed that GLS, GCS, the strains of three planes (APLAX, 4CH and 2CH) and three levels (SAX-MV, SAX-PM and SAX-AP), GSRe, and GSRs of 18 segments all significantly decreased in patients with hypercholesterolemia. In addition, LDL-c was significantly associated with the global strains, while LDL-c and EF were not associated. Taken together, these data demonstrated that although patients with hypercholesterolemia had normal EF, longitudinal and circumferential myocardial deformations decreased remarkably, while the radial strains increased.
Table VIII. Comparison of radial strain (SR) between hypercholesterolemia and control groups.

\begin{tabular}{|c|c|c|c|}
\hline SR strain $(\%)$ & $\begin{array}{l}\text { Hypercho- } \\
\text { lesterolemia } \\
\text { group }(n=47)\end{array}$ & $\begin{array}{c}\text { Control } \\
\text { group }(\mathrm{n}=37)\end{array}$ & P-value \\
\hline \multicolumn{4}{|l|}{$\begin{array}{l}\text { LAD mitral valve } \\
\text { level }\end{array}$} \\
\hline Anterior septum & $51.11 \pm 18.92$ & $40.13 \pm 23.14$ & 0.04 \\
\hline Anterior wall & $53.55 \pm 16.56$ & $40.17 \pm 24.88$ & 0.01 \\
\hline \multicolumn{4}{|l|}{$\begin{array}{l}\text { Papillary muscle } \\
\text { level }\end{array}$} \\
\hline Anterior septum & $55.19 \pm 16.61$ & $46.32 \pm 25.73$ & 0.10 \\
\hline Anterior wall & $56.54 \pm 20.95$ & $50.46 \pm 26.37$ & 0.31 \\
\hline \multicolumn{4}{|l|}{ Apical level } \\
\hline Anterior septum & $56.58 \pm 12.29$ & $41.81 \pm 19.62$ & 0.001 \\
\hline Anterior wall & $56.13 \pm 12.64$ & $44.25 \pm 22.36$ & 0.01 \\
\hline Lateral wall & $58.17 \pm 11.28$ & $53.39 \pm 22.21$ & 0.02 \\
\hline Posterior wall & $58.42 \pm 13.73$ & $49.24 \pm 12.35$ & $<0.0001$ \\
\hline Lower wall & $56.46 \pm 14.77$ & $48.48 \pm 21.29$ & $<0.0001$ \\
\hline Posterior septum & $57.71 \pm 10.32$ & $49.33 \pm 20.64$ & $<0.0001$ \\
\hline \multicolumn{4}{|l|}{$\begin{array}{l}\text { LCX mitral valve } \\
\text { level }\end{array}$} \\
\hline Lateral wall & $54.45 \pm 16.36$ & $42.27 \pm 26.18$ & 0.02 \\
\hline Lower wall & $51.89 \pm 20.51$ & $44.32 \pm 27.13$ & 0.22 \\
\hline \multicolumn{4}{|l|}{$\begin{array}{l}\text { Papillary muscle } \\
\text { level }\end{array}$} \\
\hline Lateral wall & $56.14 \pm 27.55$ & $55.66 \pm 23.57$ & 0.95 \\
\hline Lower wall & $58.68 \pm 27.49$ & $57.61 \pm 23.52$ & 0.87 \\
\hline \multicolumn{4}{|l|}{$\begin{array}{l}\text { RCA mitral valve } \\
\text { level }\end{array}$} \\
\hline Lower wall & $56.83 \pm 21.94$ & $44.65 \pm 20.96$ & 0.06 \\
\hline Posterior septum & $57.77 \pm 18.98$ & $42.79 \pm 25.81$ & 0.01 \\
\hline \multicolumn{4}{|l|}{$\begin{array}{l}\text { Papillary muscle } \\
\text { level }\end{array}$} \\
\hline Lower wall & $56.62 \pm 18.33$ & $54.84 \pm 25.35$ & 0.74 \\
\hline Posterior septum & $53.86 \pm 13.77$ & $44.81 \pm 27.29$ & 0.06 \\
\hline
\end{tabular}

LAD, left anterior descending coronary artery; LCX, left circumflex coronary artery; RCA, right coronary artery.

These findings are consistent with previous reports on manifestations of subclinical disease $(15,16)$.

An explanation for the increase of radial strains is that LV myocardial hypertrophy helped with the maintenance of normal EF in the early stage of disease. Using a mathematical model, Maciver and Townsend (17) discovered that the radial wall thickening could help maintain normal EF though the shortened long axis has undergone significant damage. Because EF remains normal even if $\mathrm{LV}$ function is damaged, EF was reported to be an insensitive index of myocardial fibrosis (18), and it is not a good index for the detection of subclinical abnormalities of systolic function (19). Kempny et al (20) found that following aortic replacement surgery, the 2-dimensional longitudinal strain of the LV wall was better than EF for predicting 


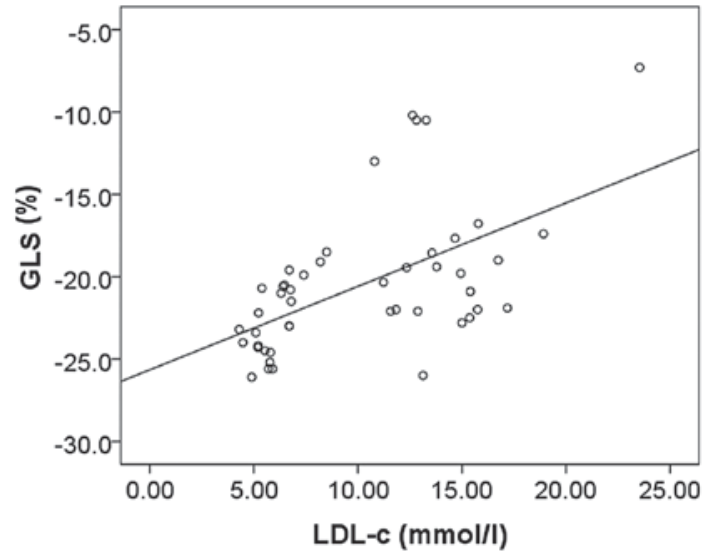

Figure 2. Correlation between global long axis strain (GLS, \%) and low-density lipoprotein cholesterol (LDL-c) in patients with hypercholesterolemia $(\mathrm{r}=0.561, \mathrm{P}=0.001)$.

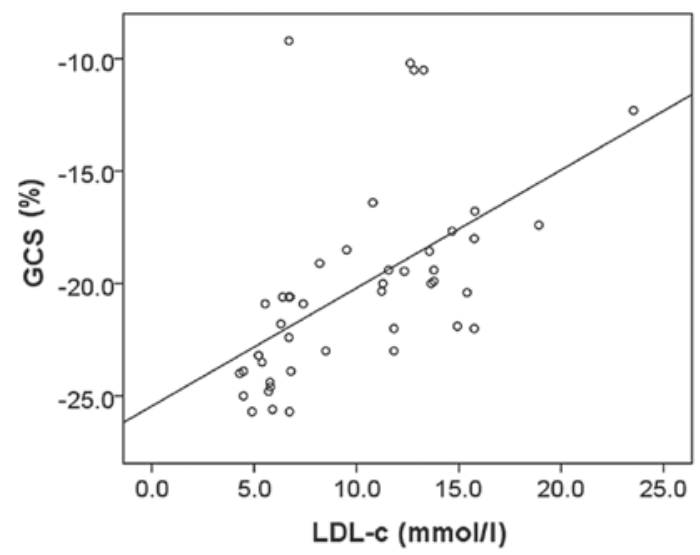

Figure 3. Correlation between global circumferential strain (GCS, \%) and low-density lipoprotein cholesterol (LDL-c) in patients with hypercholesterolemia $(r=0.565, \mathrm{P}<0.0001)$.

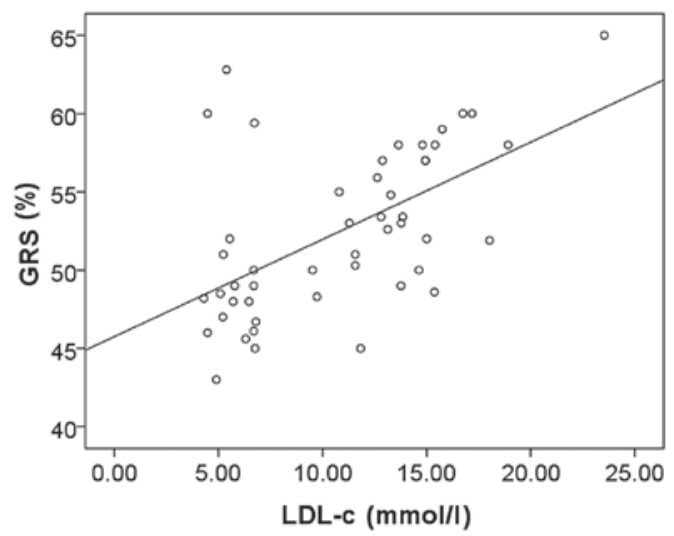

Figure 4. Correlation between global radial strain (GRS, \%) and low-density lipoprotein cholesterol (LDL-c) in patients with hypercholesterolemia $(\mathrm{r}=0.563, \mathrm{P}=0.003)$.

myocardial recovery and improvement of symptoms. It was also reported that SRs represents early myocardial systolic function, and it can reflect myocardial systolic performance more precisely than EF and SRs (21). As discussed above, EF itself is not sufficient to illustrate changes of systolic function,
Table IX. Proportion of segments with significant differences in the three main coronaries (LAD, LCX and RCA).

Segment with

significant

difference SL SC SR Proportion Result (\%)

\begin{tabular}{rrrrrl}
\hline LAD & 10 & 6 & 8 & $24 / 30$ & 80 \\
LCX & 4 & 3 & 1 & $8 / 12$ & 66.7 \\
RCA & 4 & 4 & 1 & $9 / 12$ & 75
\end{tabular}

LAD, left anterior descending coronary artery; LCX, left circumflex coronary artery; RCA, right coronary artery.

while STI is more sensitive for the diagnosis of early cardiac damage.

In this study, GSRe of APLAX, $4 \mathrm{CH}$ and $2 \mathrm{CH}$ planes, and SAX-MV and SAX-PM levels in the hypercholesterolemia group were all lower than those in the control group. There were no significant differences in GSRa and SRa of each wall segment or SRe of SAX-AP between the two groups. SRa reflects late diastolic LV strain rate at the beginning of left atrial systole, and SRe reflects LV strain rate in the rapid filling period. Early systole accounts for the majority of systole. SRe of basal and mid-segments were significantly reduced compared with those in the control group. This is because wall strain and pressure in the myocardium are highest in the endocardium, and they decrease along the epicardium (22). Hence, the longitudinal myocardium in LV endocardium is most sensitive to interstitial fibrosis and low perfusion of the microcirculation. It can be easily affected by pathological factors such as myocardial ischemia and fibrosis, which results in myocardial ischemia and fibrosis of the endocardium (23). Myocardial ischemia and fibrosis was the early damage of diastolic function of longitudinal myocardial fibers in endocardium Therefore, it was concluded that the occurrence of myocardial abnormalities in patients with hypercholesterolemia was in early diastole. SRe is a sensitive index for early prediction of diastolic dysfunction of the heart.

Because of the specialized anatomy in the apical region, the GSRe of the short axis apical plane in the two groups was not significantly different. The unique arrangement of myocardial fibers in the apical region may result in unobvious damage in the early stage of atherosclerosis. In the helical structure of the heart, myocardial arrangement in the basal and mid segments of the endocardium is parallel with the long axis, whereas apical fibers are arranged in a circle, which mainly participate in rotary motion. In this study, strain compensation in the radial direction mostly occurred in the apical region. As a result, unobvious abnormalities were induced by the excessive deposition of cholesterol in the early stage. The fact that apical abnormalities occurred later was also because of the location of atherosclerotic plaques. Plaques are able to cause blockage of the coronary artery, and are often located at the coronary ostium. Because the coronary artery is far from the apical region, apical abnormalities occur later.

Possible reasons for the characteristics of coronary artery involvement in this study were analyzed: i) Plaques tend to 
Table X. Comparison of average strains in three directions ( $\left.\operatorname{Strain}_{\mathrm{SL}, \mathrm{SC}, \mathrm{SR}}-\mathrm{Avg}\right)$ of the three main coronaries (LAD, LCX and RCA) between the hypercholesterolemia and control groups.

\begin{tabular}{lccc}
\hline Strain $(\%)$ & Hypercholesterolemia group & Control group & P-value \\
\hline LAD $^{\mathrm{a}}$ basal & $-16.19(-36.23-77.89)^{\mathrm{b}}$ & $-20.32(-39.78-68.30)$ & $<0.0001$ \\
Mid & $-17.87(-37.52-80.22)^{\mathrm{b}}$ & $-23.56(-41.07-76.06)$ & $<0.0001$ \\
Ap & $-19.39(-36.22-77.25)$ & $-24.31(-40.74-64.62)$ & $<0.0001$ \\
Average & $-17.15(-38.12-72.14)$ & $-22.97(-40.35-69.12)$ & - \\
LCX basal & $-8.98(-26.98-80.64)^{\mathrm{b}}$ & $-15.84(-31.71-81.31)$ & $<0.0001$ \\
Mid & $-10.39(-27.75-75.55)^{\mathrm{b}}$ & $-16.82(-31.49-81.64)$ & $<0.0001$ \\
Average & $-10.41(-29.41-75.9)$ & $-16.13(-31.44-81.12)$ & - \\
RCA basal & $-16.05(-36.06-89.91)^{\mathrm{b}}$ & $-19.80(-41.36-71.61)$ & 0.001 \\
Mid & $-17.96(-37.1-76.9)^{\mathrm{b}}$ & $-22.38(-42.61-84.50)$ & 0.006 \\
Average & $-17.39(-36.22-77.25)$ & $-20.91(-41.45-76.63)$ & - \\
\hline
\end{tabular}

${ }^{a}$ Three main coronaries (LAD, RCA and LCX) had significant differences $(\mathrm{P}<0.001) .{ }^{\mathrm{b}}$ Among the three coronaries, and among their basal and mid segments of patients with hypercholesterolemia, there were significant differences between LCX and LAD, and between LCX and RCA $(\mathrm{P}<0.0001)$. LAD, left anterior descending coronary artery; LCX, left circumflex coronary artery; RCA, right coronary artery.

accumulate near the main coronary artery, which results in people suffering from severe cardiac abnormalities earlier than their healthy contemporaries. Yang et al reported that the significantly reduced coronary flow reserve in patients with hypercholesterolemia was because of coronary ostial obstruction caused by the plaque (24), which was consistent with the results of the study by Neefjes et al (25). The latter reported a computed tomography study of patients with hypercholesterolemia, and confirmed that in most cases of hypercholesterolemia, the plaque is located in the center or proximity of the coronary artery; ii) the anatomical position of the LAD is higher than the LCX and the RCA. Therefore, blood flows through the LAD earlier than the other two coronary arteries. According to the 16-segment or 17-segment model issued by the ASE, the LAD accounts for the majority of myocardial segments. Therefore, myocardial deformation mainly occurs in the LAD; and iii) the anatomy of the LCX is such that it protrudes at a $90^{\circ}$ angle from the left main coronary artery. In addition, the LCX is smaller in diameter, and its range of distribution is smaller than that of the other two vessels. Therefore, this vessel has the tendency to become severely damaged once it is blocked by plaques.

It was reported that early and intensive treatment before the appearance of clinical symptoms can help patients with hypercholesterolemia achieve the same life expectancy as their contemporaries (26). However, most patients with hypercholesterolemia across the world have not been diagnosed or treated. Moreover, it is fairly difficult to reduce cholesterol to target levels by combination therapy. Therefore, it is expected that the emergence of effective clinical tools such as STI will provide physicians with useful information related to hypercholesterolemia to achieve early diagnosis and effective treatment.

\section{References}

1. Seo Y, Ishizu T, Enomoto Y, Sugimori H, Yamamoto M, Machino T, Kawamura R, Aonuma K: Validation of 3-dimensional speckle tracking imaging to quantify regional myocardial deformation. Circ Cardiovasc Imaging 2: 451-459, 2009.
2. Takeuchi M, Nishikage T, Nakai H, Kokumai M, Otani S and Lang RM: The assessment of left ventricular twist in anterior wall myocardial infarction using two-dimensional speckle tracking imaging. J Am Soc Echocardiogr 20: 36-44, 2007.

3. Leung DY and Ng AC: Emerging clinical role of strain imaging in echocardiography. Heart Lung Circ 19: 161-174, 2010.

4. Gervas J and Pérez Fernández M: Diagnosis of hypercholesterolemia. Aten Primaria 17: 231-232, 1996 (In Spanish).

5. Schiller NB, Shah PM, Crawford M, DeMaria A, Devereux R, Feigenbaum H, Gutgesell H, Reichek N, Sahn D, Schnittger I, et al: Recommendations for quantitation of the left ventricle by two-dimensional echocardiography. American Society of Echocardiography Committee on Standards, Subcommittee on Quantitation of Two-Dimensional Echocardiograms. J Am Soc Echocardiogr 2: 358-367, 1989.

6. Liyanage KE, Burnett JR, Hooper AJ and van Bockxmeer FM: Familial hypercholesterolemia: epidemiology, Neolithic origins and modern geographic distribution. Crit Rev Clin Lab Sci 48: 1-18, 2011.

7. Hopkins PN, Toth PP, Ballantyne CM and Rader DJ; National Lipid Association Expert Panel on Familial Hypercholesterolemia: Familial hypercholesterolemias: prevalence, genetics, diagnosis and screening recommendations from the National Lipid Association Expert Panel on Familial Hypercholesterolemia. J Clin Lipidol 5 (Suppl 3): S9-S17, 2011.

8. Nordestgaard BG, Chapman MJ, Humphries SE, Ginsberg HN, Masana L, Descamps OS, Wiklund O, Hegele RA, Raal FJ, Defesche JC, et al; European Atherosclerosis Society Consensus Panel: Familial hypercholesterolaemia is underdiagnosed and undertreated in the general population: guidance for clinicians to prevent coronary heart disease: consensus statement of the European Atherosclerosis Society. Eur Heart J 34: 3478a-3490a, 2013.

9. Al-Sarraf A, Allard M, Martinka M and Frohlich J: Regional and national familial hypercholesterolemia registries: present international application, importance, and needs for Canada. Can J Cardiol 29: 6-9, 2013.

10. Muranaka A, Yuda S, Tsuchihashi K, Hashimoto A, Nakata T, Miura T, Tsuzuki M, Wakabayashi C, Watanabe $\mathrm{N}$ and Shimamoto K: Quantitative assessment of left ventricular and left atrial functions by strain rate imaging in diabetic patients with and without hypertension. Echocardiography 26: 262-271, 2009.

11. Koyama J, Ray-Sequin PA and Falk RH: Longitudinal myocardial function assessed by tissue velocity, strain, and strain rate tissue Doppler echocardiography in patients with AL (primary) cardiac amyloidosis. Circulation 107: 2446-2452, 2003.

12. Weidemann F, Eyskens B, Mertens L, Di Salvo G, Strotmann J, Buyse G, Claus P, D'hooge J, Bijnens B, Gewillig M, et al: Quantification of regional right and left ventricular function by ultrasonic strain rate and strain indexes in Friedreich's ataxia. Am J Cardiol 91: 622-626, 2003. 
13. Jurcut R, Wildiers H, Ganame J, D'hooge J, De Backer J, Denys H, Paridaens R, Rademakers F and Voigt JU: Strain rate imaging detects early cardiac effects of pegylated liposomal Doxorubicin as adjuvant therapy in elderly patients with breast cancer. J Am Soc Echocardiogr 21: 1283-1289, 2008.

14. Sitia S, Tomasoni L, Cicala S, Atzeni F, Ricci C, Gaeta M, Sarzi-Puttini P and Turiel M: Detection of preclinical impairment of myocardial function in rheumatoid arthritis patients with short disease duration by speckle tracking echocardiography. Int J Cardiol 160: 8-14, 2012

15. Fang ZY, Leano R and Marwick TH: Relationship between longitudinal and radial contractility in subclinical diabetic heart disease. Clin Sci (Lond) 106: 53-60, 2004.

16. Di Salvo G, D'Aiello AF, Castaldi B, Fadel B, Limongelli G, D'Andrea A, Pergola V, Pacileo G, Del Giudice EM, Perrone L, et al: Early left ventricular abnormalities in children with heterozygous familial hypercholesterolemia. J Am Soc Echocardiogr 25: 1075-1082, 2012.

17. Maciver DH and Townsend M: A novel mechanism of heart failure with normal ejection fraction. Heart 94: 446-449, 2008.

18. Weidemann F, Herrmann S, Störk S, Niemann M, Frantz S Lange V, Beer M, Gattenlöhner S, Voelker W, Ertl G, et al: Impact of myocardial fibrosis in patients with symptomatic severe aortic stenosis. Circulation 120: 577-584, 2009.

19. Nakai H, Takeuchi M, Nishikage T, Lang RM and Otsuji Y: Subclinical left ventricular dysfunction in asymptomatic diabetic patients assessed by two-dimensional speckle tracking echocardiography: correlation with diabetic duration. Eur J Echocardiogr 10: 926-932, 2009.

20. Kempny A, Diller GP, Kaleschke G, Orwat S, Funke A, Radke R, Schmidt R, Kerckhoff G, Ghezelbash F, Rukosujew A, et al: Longitudinal left ventricular 2D strain is superior to ejection fraction in predicting myocardial recovery and symptomatic improvement after aortic valve implantation. Int J Cardiol 167: 2239-2243, 2013.
21. Stoylen A, Heimdal A, Bjornstad K, Torp HG and Skjaerpe T: Strain rate imaging by ultrasound in the diagnosis of regional dysfunction of the left ventricle. Echocardiography 16: 321-329, 1999.

22. Pavlopoulos $\mathrm{H}$ and Nihoyannopoulos P: Abnormal segmental relaxation patterns in hypertensive disease and symptomatic diastolic dysfunction detected by strain echocardiography. J Am Soc Echocardiogr 21: 899-906, 2008.

23. Youn HJ, Ihm SH, Lee JM, Park CS, Cho EJ, Jung HO, Jeon HK, Oh YS, Chung WS, Kim JH, et al: Relation between flow reserve capacity of penetrating intramyocardial coronary arteries and myocardial fibrosis in hypertension: study using transthoracic Doppler echocardiography. J Am Soc Echocardiogr 19: 373-378, 2006.

24. Yang Y, Zhang X, Li R, Ren H, Wang Z, Li Z, Lin J, Wang L, $\mathrm{Yu} \mathrm{W}$ and Zhang Z: Evaluation of coronary flow velocity reserve in homozygous familial hypercholesterolemia by transthoracic Doppler echocardiography and dual-source computed tomography. Ultrasound Med Biol 36: 1756-1761, 2010.

25. Neefjes LA, Ten Kate GJ, Alexia R, Nieman K, Galema-Boers AJ, Langendonk JG, Weustink AC, Mollet NR, Sijbrands EJ, Krestin GP, et al: Accelerated subclinical coronary atherosclerosis in patients with familial hypercholesterolemia. Atherosclerosis 219: 721-727, 2011

26. Robinson JG and Goldberg AC; National Lipid Association Expert Panel on Familial Hypercholesterolemia: Treatment of adults with familial hypercholesterolemia and evidence for treatment: recommendations from the National Lipid Association Expert Panel on Familial Hypercholesterolemia. J Clin Lipidol 5 (Suppl 3): S18-S29, 2011. 